\title{
Fisiopatologia e trattamento dell'iperparatiroidismo primario
}

\author{
C. Marcocci, C. Banti, F. Cetani \\ Dipartimento di Endocrinologia, Università di Pisa, Pisa
}

\section{Fisiopatologia}

Liperparatiroidismo primario (IPTP) è causato nell' $85 \%$ dei casi da un adenoma singolo, nel 10\% da iperplasia e nello $0.5-1 \%$ da carcinoma delle paratiroidi (1). Gli studi di genetica molecolare hanno contribuito negli ultimi anni a chiarire alcune alterazioni geniche alla base della tumorigenesi paratiroidea. La fisiopatologia dell'IPTP può essere brevemente riassunta come segue:

1. Disordine del "set-point" calcio-dipendente che determina la perdita del normale controllo a feedback sulla produzione e secrezione del PTH esercitato dalla concentrazione extracellulare del calcio (2).

2. Disordine della proliferazione cellulare causata da alterazioni di diversi meccanismi genetici con perdita o guadagno clonale di funzione/i cellulare/i, come chiaramente dimostrato da studi di genetica molecolare (3).

Per quanto riguarda le alterazioni del set-point in condizioni normali il calcio sierico costituisce il principale regolatore della secrezione di PTH (3). In effetti le condizioni che determinano anche un piccolo incremento del calcio sierico provocano una significativa inibizione della secrezione di PTH. Questo si verifica perché un incremento del calcio extracellulare determina l'attivazione del recettore del calcio (Calcium Sensing Receptor, CaSR) (2), provocando una cascata intracellulare fosfolipasi $\mathrm{C}$ dipendente che porta all'aumento dei livelli di inositolo trifosfato i quali a loro volta agiscono sul reticolo endoplasmatico favorendo la liberazione di calcio intracellulare. Quest'ultimo agisce come secondo messaggero provocando una degradazione delle vescicole secretorie di PTH con riduzione della sua secrezione (Fig. 1). Il CaSR è un re- cettore di membrana e appartiene alla superfamiglia dei recettori accoppiati alle proteine G. Esso è costituito da 3 domini: una grande porzione extracellulare che lega il ligando, una porzione transmembrana costituita da sette domini e una porzione intracellulare che costituisce l'effettore del recettore attraverso l'accoppiamento con la proteina G (Fig. 2). Il CaSR è espresso principalmente sulla superficie delle cellule paratiroidee e renali, ma è presente anche in numerosi altri tessuti tra cui intestino, osso, cartilagine e in molti altri ancora. I suoi effetti sono noti per quanto riguarda il

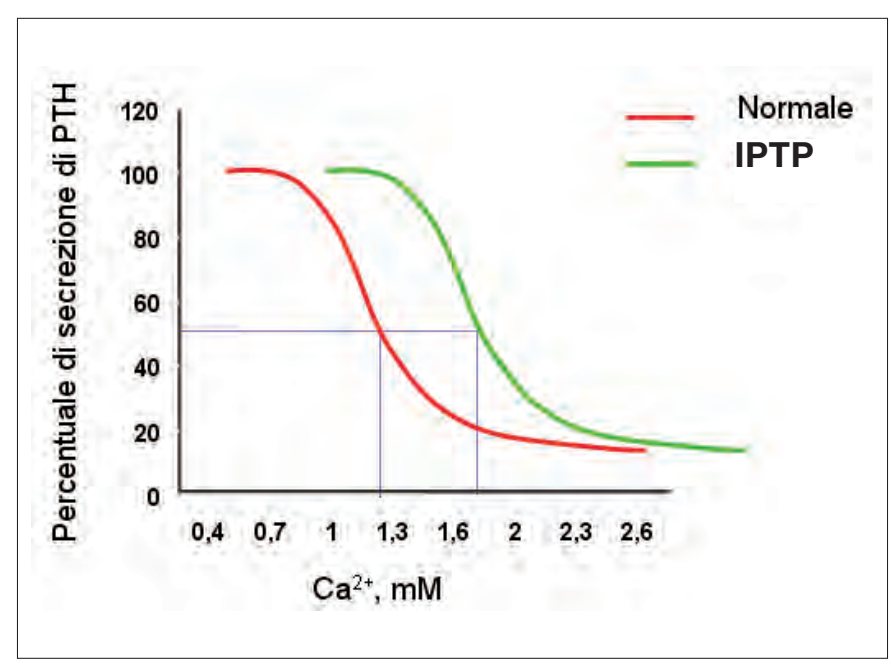

Fig. 1 - Il "set-point" del calcio rappresenta quella concentrazione di calcio per la quale la secrezione di PTH si riduce del $50 \%$. Nell'IPTP questa curva si sposta a destra per cui sono necessarie concentrazioni maggiori di calcio per ottenere l'inibizione del $\mathbf{5 0} \%$ del PTH. 


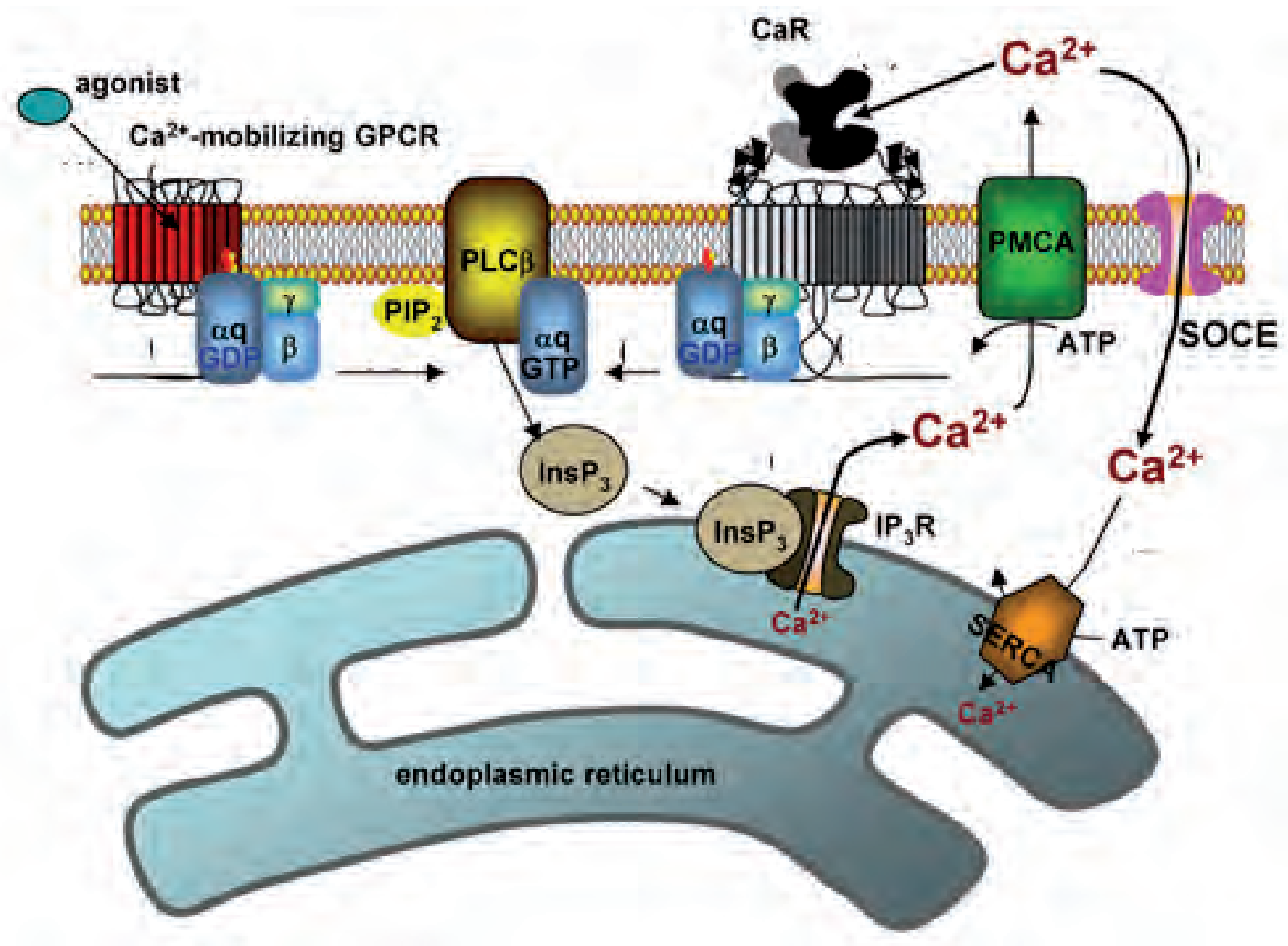

Fig. 2 - Modificata da Breitwieser G.E. "Extracellular Calcium as an integrator of tissue function". Int J Biochem Cell Biol 2008. Il CaSR è espresso sulla superficie delle cellule paratiroidee II calcio extracellulare agisce sul controllo della secrezione di PTH attraverso l'attivazione del CaSR, provocando una cascata intracellulare che porta all'aumento dei livelli di inositolo trifosfato e della fosfolipasi $\mathrm{C}$ che a loro volta agiscono sul reticolo endocitoplasmatico favorendo la liberazione di calcio intracellulare il quale agisce come secondo messaggero e provoca una degradazione di vescicole secretorie di PTH.

rene e l'osso, ma ancora da chiarire per quanto riguarda le azioni su altri organi.

Nell'IPTP si verifica una alterazione del "set-point" poiché le cellule dell'adenoma paratiroideo hanno una riduzione della loro sensibilità di rispondere alle concentrazioni di calcio extracellulare (2). Pertanto è necessaria una concentrazione maggiore di calcio per inibire la secrezione del PTH. Nell'IPTP da iperplasia non si verifica una alterazione del set-point delle cellule paratiroidee, bensi l'incremento del numero delle cellule stesse provoca l'insorgenza dell'ipercalcemia.

Per quanto riguarda le alterazioni della proliferazione cellulare studi genetici hanno evidenziato il coinvolgimento sia di oncogeni che di geni oncosoppressori (1). La dimostrazione dell'origine clonale della maggior parte degli adenomi suggerisce che vi sia un difetto nei geni che controllano la crescita cellulare o l'espressione del PTH. Nel 20-40\% degli adenomi di pazienti con IPTP è stato dimostrato un riarrangiamento del gene del PTH (localizzato sul baccio corto del cromosoma 11) con traslocazione pericentromerica della sequenza promotrice del gene del PTH che si pone a monte del proto-oncogene PRAD 1, codificante per la ciclina D1. Si determina così un aumento dell'espressione di quest'ultima con alterata regolazione del ciclo cellulare. Anche il gene oncosoppressore MEN1, localizzato sul cromosoma 11, il quale codifica per la menina risulta essere implicato nella patogenesi degli adenomi delle paratiroidi (in circa il 15-20\% degli adenomi) (1). Alterazioni dell'espressione di altri geni quali CaSR e il recettore per la vitamina $\mathrm{D}$ sono state identificate, ma rimane da chiarire quale sia il loro ruolo patogenetico (1). 


\section{Trattamento}

\section{Terapia chirurgica}

L'intervento chirurgico rappresenta la terapia di scelta nei pazienti affetti da IPTP. Le indicazioni al trattamento chirurgico sono state recentemente riviste e riassunte nella Tabella I (4). Tuttavia, la terapia medica può essere presa in considerazione nei seguenti casi: a) pazienti che rifiutano la chirurgia; b) presenza di malattie concomitanti gravi che controindicano l'intervento chirurgico; c) pazienti con IPTP nei quali la paratiroidectomia sarebbe indicata sulla base dei valori sierici di calcio (in accordo con le relative linee guida di trattamento), ma nei quali l'intervento chirurgico non è clinicamente appropriato o è controindicato; d) impossibilità di reperire un chirurgo esperto; e) IPTP persistente dopo ripetuti interventi chirurgici; f) carcinoma paratiroideo inoperabile.

\section{Osservazione e monitoraggio}

Nei pazienti nei quali non sussistono i criteri per l'intervento è raccomandato un periodico monitoraggio eventualmente associato, in casi selezionati, ad un trat-

\section{TABELLA I - CRITERI PER IL TRATTAMENTO CHIRURGI-} CO DELL'IPTP ASINTOMATICO

- Calcemia: valori al di sopra di $1 \mathrm{mg} / \mathrm{dL}$ rispetto al limite superiore della norma

- Clearance creatinina inferiore a $60 \mathrm{~mL} / \mathrm{min}$

- Densità minerale ossea: T-score* $<-2.5$ (qualunque sito) e/o precedente frattura di fragilità

- Età $<50$ anni

* il T score equivale alla deviazione standard della media dei valori di riferimento (soggetti normali dello stesso sesso con età compresa tra 20 e 40 anni). Un valore di T score $<-2.5$ corrisponde a una densità minerale ossea inferiore alla media meno 2.5 deviazione standard dei controlli

TABELLA II - MONITORAGGIO DELLIPTP ASINTOMATICO NON TRATTATO CHIRURGICAMENTE

- Calcemia: ogni anno

- Creatininemia: ogni anno *

- Densità minerale ossea: ogni 1-2 anni (tutti i 3 siti)

* per il calcolo della clearance della creatinina si raccomanda l'uso dell'equazione MDRD n. 7 (Modification of Diet in Renal Disease Study) tamento medico (Tab. II) (5). Diversi studi hanno documentato una sostanziale stabilità del quadro clinico nei pazienti con IPTP lieve, che non soddisfano i criteri chirurgici (6-7). Il paziente dovrà essere indirizzato allintervento laddove, nel corso del follow-up, siano soddisfatti uno o più criteri per l'intervento chirurgico. Uno studio di follow-up a lungo termine fino a 15 anni ha documentato che tale evenienza può accadere in circa il $40 \%$ dei casi (7) .

\section{Terapia medica}

Per quanto concerne il trattamento medico dell'IPTP i farmaci usati possono essere divisi in: calciomimetici e anticatabolici.

\section{Calciomimetici}

I farmaci calciomimetici sono in grado di legarsi, come il calcio ionizzato, alla porzione extracellulare del CaSR determinando un aumento della sensibilità del recettore stesso al calcio ionizzato; l'effetto prodotto è quello di una inibizione della secrezione di PTH. Pertanto, tali farmaci forniscono una opzione terapeutica nell'IPTP. L'unico calciomimetico disponibile è il Cinacalcet. Questo farmaco è stato utilizzato nei pazienti con IPTP da carcinoma paratiroideo recidivante/metastatico nei quali ha dimostrato una efficacia parziale e limitata nel tempo nel controllo dell'ipercalcemia. Recentemente, il Cinacalcet è stato approvato per il trattamento dei pazienti con IPTP nei quali la paratiroidectomia sarebbe indicata sulla base dei valori sierici di calcio (in accordo con le relative linee guida di trattamento), ma nei quali l'intervento chirurgico non è clinicamente appropriato o è controindicato (8). Uno studio randomizzato a breve termine condotto da Shoback (9) in pazienti con IPTP, ha dimostrato che il Cinacalcet normalizza i livelli di calcio e riduce, seppur modicamente, i livelli di PTH. Questi risultati sono stati confermati in uno studio a lungo termine randomizzato in doppio cieco condotto da Peacock su pazienti con IPTP con ipercalcemia lieve o moderata $(>10.3-$ $<12.5 \mathrm{mg} / \mathrm{dL}$ ) (10). In questo studio sono stati arruolati 78 pazienti trattati con Cinacalcet $(30-50 \mathrm{mg}$, due volte al giorno) per 52 settimane. L'obiettivo primario dello studio era il raggiungimento della normocalcemia (valori medi di calcio $\leq 10.3 \mathrm{mg} / \mathrm{dL}$ ) con una riduzione della calcemia di almeno $0.5 \mathrm{mg} / \mathrm{dL}$ rispetto al valore basale. Gli obiettivi secondari includevano le modificazioni dei parametri biochimici e urinari e della densitometria ossea (BMD). Il 73\% dei pazienti trattati 
con Cinacalcet raggiungevano l'obiettivo primario $(\mathrm{p}<0.001)$. I livelli di calcemia si normalizzavano entro le prime due settimane nel $90 \%$ dei pazienti che assumevano la dose più bassa di Cinacalcet $(30 \mathrm{mg})$ senza nessuna modificazione significativa nel gruppo placebo. I livelli circolanti di PTH diminuivano del 7.6\% nei pazienti trattati con Cinacalcet ma aumentavano del $7.7 \%$ nei pazienti del gruppo di controllo $(\mathrm{p}<0.01)$. Dopo 52 settimane, i marcatori di riassorbimento e neoformazione ossea aumentavano nei pazienti trattati $(\mathrm{p}<0.05)$, rimanendo nel range della norma senza modificazioni della BMD. I risultati di questo ultimo studio sono stati confermati in una sua estensione a 3 anni (11).

\section{Anticatabolici}

I farmaci anticatabolici sono rappresentati da bisfosfonati, estrogeni e raloxifene. Essi agiscono attraverso l'inibizione degli osteoclasti, determinando una riduzione dell'efflusso di calcio dallo scheletro, determinando una lieve riduzione dei livelli di calcio e della calciuria e prevenendo la perdita di massa ossea; tuttavia, i livelli di PTH possono aumentare durante il trattamento con bisfosfonati.

\section{Estrogeni}

Esiste un solo studio randomizzato che dimostra un aumento significativo della BMD a livello lombare $(+5.2 \%)$ e del collo femorale $(+3.4 \%)$. Tuttavia alla luce dei noti effetti collaterali della terapia estrogenica ed in particolare del possibile effetto additivo sul rischio cardiovascolare, già aumentato nei pazienti con IPTP (12), l'uso a lungo termine della terapia estrogenica in questi pazienti non è raccomandato.

\section{Raloxifene}

Il raloxifene, modulatore selettivo del recettore degli estrogeni, agendo come agonista a livello osseo determina gli stessi effetti antiriassorbitivi sul metabolismo minerale nelle donne in postmenopausa affette da IPTP. Il trattamento a breve termine (8 settimane) con raloxifene determina una riduzione significativa della calcemia e dei marker di rimodellamento osseo, che tornano ai valori basali dopo sospensione del trattamento, mentre non si osservano variazioni dei livelli di PTH (13). Uno studio condotto in 3 donne osteopeniche postmenopausali con IPTP asintomatico trattate per 1 anno ha dimostrato una riduzione della calcemia e transitoria del PTH, ed un aumento della BMD lombare $(+3.4 \%)$ e del collo femorale $(+2.4 \%)$ (14). Tuttavia al momento non sono disponibili dati di efficacia a lungo termine.

\section{Bisfosfonati}

I bisfosfonati inibiscono gli osteoclasti legandosi ai cristalli di idrossiapatite della matrice ossea agendo da potenti inibitori del riassorbimento osseo. Vari bisfosfonati (BF) sono stati impiegati nella terapia dell'IPTP sia per via orale che endovenosa. L'alendronato (15-16) è stato utilizzato per oltre 1 anno ed in studi randomizzati. Tale farmaco somministrato per via orale non determina variazioni significative della calcemia, ma si accompagna ad una tendenza all'incremento dei livelli di PTH. I marker di rimodellamento osseo si riducono costantemente. Tutti gli studi documentano un aumento della BMD a livello lombare e femorale, ma non radiale.

\section{Considerazioni conclusive}

Al momento non esistono farmaci ideali per il trattamento dell'IPTP. I farmaci anticatabolici (bisfosfonati e raloxifene) determinano una lieve riduzione dei livelli di calcio e prevengono la perdita di massa ossea; tuttavia, i livelli di PTH possono aumentare durante il trattamento con bisfosfonati. Il cinacalcet riduce la calcemia ed il $\mathrm{PTH}$, ma non ha effetti sulla BMD. Pertanto, i calciomimetici possono costituire una valida alternativa nei pazienti con IPTP lieve non associato ad osteoporosi, in virtù del possibile beneficio sulle manifestazioni neuropsichiche e cognitive, conseguente alla riduzione della calcemia; ed inoltre essi rappresentano un utile presidio nei pazienti con carcinoma paratiroideo non aggredibile chirurgicamente.

Va tuttavia rilevato come il trattamento medico dell'IPTP, basato sulla inibizione sia della secrezione del PTH che dell'effetto a carico degli organi bersaglio, non sia risolutivo e pertanto richieda una somministrazione cronica, eventualmente anche a vita e pertanto va infine valutato in termini di costo-efficacia il rapporto tra terapia medica e terapia chirurgica. Le raccomandazioni definitive per il trattamento medico a lungo termine dell'IPTP devono attendere studi prospettici ben controllati con terapie mediche specifiche confrontate con la chirurgia. Lo sviluppo di un potente calciomimetico a lunga azione, l'applicazione clinica di specifici antagonisti del 
PTH e analoghi della vitamina D non ipercalcemizzanti (in grado però di inibire la secrezione di PTH) e lo sviluppo di farmaci potenti che bloccano l'azione del PTH a diversi livelli è di grande auspicio per un approccio futuro alla terapia medica dell'IPTP.
Indirizzo degli Autori:

Claudio Marcocci, MD

Dipartimento di Endocrinologia

Università di Pisa

Via Paradisa, 2

56124 Pisa

c.marcocci@endoc.med.unipi.it

\section{Bibliografia}

1. Arnold A, Shatttuck TM, Mallya SM, et al. Molecular pathogenesis of primary hyperparathyroidism. J Bone Miner Res 2002; (Suppl 2): S30-6.

2. Brown EM. Four-parameter model of the sigmoidal relationship between parathyroid hormone release and extracellular calcium concentration in normal and abnormal parathyroid tissue. J Clin Endocrinol Metab 1983, 56: 572-81.

3. Brown EM. The calcium-sensing receptor: physiology, pathophysiology and CaR-based therapeutics. Subcell Biochem 2007; 45:139-67.

4. Bilezikian JP, Khan AA, Potts JT Jr. Third International Workshop on the Management of Asymptomatic Primary Hyperthyroidism. Guidelines for the management of asymptomatic primary hyperparathyroidism: summary statement from the third international workshop. J Clin Endocrinol Metab 2009; 94: 335-9.

5. Khan A, Grey A, Shoback D. Medical management of asymptomatic primary hyperparathyroidism: proceedings of the third international workshop. J Clin Endocrinol Metab 2009; 94: 373-81.

6. Silverberg JP, Shane E, Jacobs TP, Siris ES, Bilezikian JP. A ten-year prospective study of primary hyperparathyroidism with or without parathyroid surgery, N Engl J Med 1999; 341: 1249-555.

7. Rubin MR, Bilezikian JP, McMahon DJ, et al. The natural history of primary hyperparathyroidism with or without parathyroid surgery after 15 years. J Clin Endocrinol Metab 2008; 93: 3462-70.

8. Gazzetta Ufficiale del 01-10-2008, 230.

9. Shoback D, Bilezikian JP, Turner SA, McCary LC,
Guo MD, Peacock M. The calcimimetic cinacalcet normalizes serum calcium in subjects with primary hyperparathyroidism. J Endocrinol Metab 2003; 88: 5644-9.

10. Peacock M, Bilezikian JP, Klassen PS, Guo MD, Turner SA, Shoback D. Cinacalcet hydrochoride maintains long-term normocalcemia in patients with primary hyperparathyroidism. J Clin Endocrinol Metab 2005; 90: 35-41.

11. Peacock M, Scumpia S, Bolognese MA, et al. Longterm control of primary hyperparathyroidism with cinacalcet. J Bone Miner Res 2006; 21 (Suppl 1): S38.

12. Grey AB, Stapleton JP, Evans MC, Tatnell M, Reid IR. Effect of hormone replacement therapy on bone mineral density in postmenopausal women with mild primary hyperparathyroidism. Ann Int Med 1996; 125: 360-8.

13. Rubin MR, Lee KH, McMahon DJ, Silverberg SJ. Raloxifene lowers serum calcium and markers of bone turnover in postmenopausal women with primary hyperparathyroidism. J Clin Endocrinol Metab 2003; 88: 1174-8.

14. Zanchetta JR, Bogado C. Raloxifene reverses bone loss in postmenopausal women with mild asymptomatic primary hyperparathyroidism. J Bone Miner Res 2001; 1: 189-90.

15. Rossini M, Gatti D, Isaia G, Sartori L, Braga V, Adami S. Effects of oral alendronate in elderly patients with osteoporosis and mild primary hyperparathyroidism. J Bone Miner Res 2001; 16: 113-9.

16. Khan AA., Bilezikian JP, Kung AWC, et al. Alendronate in primary hyperparathyroidism: a doubleblind, randomized, placebo-controlled trial. J Endocrinol Metab 2004; 89: 3319-25. 